Notre Dame Journal of Formal Logic

Volume 48, Number 1, 2007

\title{
Complexity Ranks of Countable Models
}

\author{
Su Gao
}

\begin{abstract}
We define some variations of the Scott rank for countable models and obtain some inequalities involving the ranks. For mono-unary algebras we prove that the game rank of any subtree does not exceed the game rank of the whole model. However, similar questions about linear orders remain unresolved.
\end{abstract}

\section{Introduction and Motivation}

Vaught's Conjecture is a dichotomy statement about the isomorphism relation of countable models. From the point of view of descriptive set theory of equivalence relations, there are stronger dichotomy statements which provide more information about the isomorphism relation. These dichotomies are usually formulated in terms of Borel reducibility. For a definition of Borel reducibility and of the equivalence relations mentioned in the rest of this introduction the reader can consult [2].

The following three dichotomy theorems form a sequence of ever stronger dichotomies. We will need the following notation. Let $\mathcal{L}$ be a countable language and $\sigma \in \mathscr{L}_{\omega_{1} \omega}$ be a sentence. Let $\operatorname{Mod}(\sigma)$ denote all countable models of $\sigma$ with universe $\omega$. Let $\cong_{\sigma}$ denote the isomorphism relation on $\operatorname{Mod}(\sigma)$.

Theorem 1.1 (Vaught's Conjecture/Silver dichotomy: Silver, cf. [7]) If $\cong_{\sigma}$ is Borel, then exactly one of the following holds:

(i) $\cong_{\sigma} \leq_{B} \operatorname{id}(\mathbb{N})$;

(ii) $\operatorname{id}(\mathbb{R}) \leq_{B} \cong_{\sigma}$.

Theorem 1.2 (Glimm-Effros dichotomy: Harrington-Kechris-Louveau [5]) If $\cong_{\sigma}$ is Borel, then exactly one of the following holds:

(i) $\cong_{\sigma} \leq_{B} \operatorname{id}(\mathbb{R})$;

(ii) $E_{0} \leq_{B} \cong_{\sigma}$.

Received February 24, 2006; accepted July 21, 2006; printed February 26, 2007

2000 Mathematics Subject Classification: Primary, 03C15, 03C64, 03 E15

Keywords: isomorphism relation, Scott rank, mono-unary algebras

(c)2007 University of Notre Dame 
Theorem 1.3 (Hjorth [6]) If $\cong_{\sigma}$ is Borel, then exactly one of the following holds:

(i) $\cong_{\sigma} \leq_{B} E_{\infty}\left(\cong_{\sigma}\right.$ is essentially countable);

(ii) $\left(E_{0}\right)^{\omega} \leq_{B} \cong_{\sigma}$.

In the above, only the third theorem is not known to hold in a more general context. The Silver dichotomy is true for all $\Pi_{1}^{1}$ equivalence relations (not necessarily isomorphism relations, not necessarily Borel). The Glimm-Effros dichotomy is true for all Borel equivalence relations (not necessarily isomorphism relations).

On the other hand, without assuming the isomorphism relation to be Borel, the $\mathcal{L}_{\omega_{1} \omega}$ Vaught's Conjecture is known to be true for a number of classes of models. We mention one here.

Theorem 1.4 (Steel [13]) Let $\mathcal{L}=\{<\}$ and $\sigma \in \mathcal{L}_{\omega_{1} \omega}$ be a sentence all of whose models are trees; that is, if $M \models \sigma$ and $a \in M$ then $\{b \in M \mid b<a\}$ is linearly ordered by $<$. Then Vaught's Conjecture holds for $\operatorname{Mod}(\sigma)$.

The trees considered by Steel are very general. In particular, they include all linear orders and mono-unary algebras (models in the language of one unary function), whereas the usual trees studied in descriptive set theory (rooted graph-theoretical trees) essentially form only a subclass of mono-unary algebras. Prior to Steel's result Vaught's conjecture for first-order theories of mono-unary algebras had been established by Marcus [10] and Miller [11], and the $\mathscr{L}_{\omega_{1} \omega}$ Vaught's Conjecture for linear orders had been proved by Rubin [12]. Then there were some further dichotomies in the special cases of linear orders and mono-unary algebras.

Theorem 1.5 ([4]) Let $\mathcal{L}=\{<\}$ and $\sigma \in \mathcal{L}_{\omega_{1} \omega}$ be a sentence all of whose models are linear orders. Then the Glimm-Effros dichotomy holds for $\operatorname{Mod}(\sigma)$.

Theorem 1.6 ([3]) Let $\mathcal{L}=\{f\}$, where $f$ is a unary function symbol, and $\sigma \in \mathcal{L}_{\omega_{1} \omega}$ be a sentence. Then exactly one of the following holds:

(i) $\cong_{\sigma}$ is Borel;

(ii) the isomorphism relation of all countable graphs is Borel reducible to $\cong_{\sigma}$.

The former group of results was proved using Gandy-Harrington forcing. The proofs for the latter group of results rely on connections to model theory of $\mathcal{L}_{\omega_{1} \omega}$. Specifically, detailed analysis of countable models following Scott (cf. [1]) allows us to gain understanding of the countable models in question in a fundamental way. Then this understanding of individual models can be gathered to show global results about the isomorphism relation. The following theorem is a manifestation of this connection between the global and local analysis of countable models.

Theorem 1.7 (Sacks, cf. [9] and [13]) Let $\mathcal{L}$ be an arbitrary countable language and $\sigma \in \mathcal{L}_{\omega_{1} \omega}$ be a sentence. Suppose there is $\alpha<\omega_{1}$ such that for any countable model $M$ of $\sigma$, whenever $\operatorname{sr}(M) \geq \alpha, \operatorname{sr}(M)<\omega_{1}^{\mathrm{CK}(M)}$. Then $\cong_{\sigma}$ is Borel.

Thus the Scott analysis, especially the computation of Scott ranks, is not only interesting in its own right, but also instrumental in the proofs of most of the theorems about the isomorphism relation, and even more so for concrete classes of models, since the focus on a specific kind of models usually brings about definite and accurate results. This is the motivation for our study of some variations of the Scott rank in the rest of this article. The rationale is that the more we understand the computation of these ranks, the better we understand the models as well as the isomorphism relation. 


\section{The Scott Analysis and the Scott Rank}

For the convenience of the reader and also to fix some notation, we recall some details of the Scott analysis here. Let $\mathcal{L}$ be a countable relational language. For countable $\mathcal{L}$-models $M, N$, and ordinal $\alpha<\omega_{1}$, the $\alpha$-equivalence between $M$ and $N$, denoted $M \equiv_{\alpha} N$, is defined by induction on $\alpha<\omega_{1}$ as follows:

(i) $M \equiv_{0} N$ iff $\operatorname{Diag}(M)=\operatorname{Diag}(N)$, where $\operatorname{Diag}(M)$ is the set of all quantifierfree $\mathcal{L}$-sentences $\varphi$ such that $M \models \varphi$;

(ii) for a limit $\lambda, M \equiv_{\lambda} N$ iff $M \equiv_{\beta} N$ for all $\beta<\lambda$;

(iii) $M \equiv_{\alpha+1} N$ iff $\forall a \in|M| \exists b \in|N|(M, a) \equiv_{\alpha}(N, b)$ and $\forall b \in|N| \exists a \in$ $|M|(M, a) \equiv_{\alpha}(N, b)$, where $(M, a),(N, b)$ are considered as $\mathcal{L}^{\prime}$-models with $\mathcal{L}^{\prime}=\mathcal{L} \cup\{c\}$ where $c$ is a fresh constant symbol.

Note that the above definition requires that $\mathcal{L}$ is a relational language; $M$ and $N$ are countable $\mathcal{L}$-models and $\alpha<\omega_{1}$. However, in practice we will deal with languages with function symbols; in doing this we simply represent each function symbol by a binary relation symbol in the obvious way. Also the notion of $\alpha$-equivalence makes sense for arbitrary ordinal $\alpha$ and for models $M$ and $N$ of arbitrary size. Nevertheless, we never consider uncountable models and uncountable ordinals in this article.

The notion of $\alpha$-equivalence can be directly captured by infinitary formulas in $\mathcal{L}_{\omega_{1} \omega}$. Given an $\mathcal{L}$-model $M$ and a tuple $\vec{a} \in|M|^{n}$ for some $n \in \omega$, the canonical Scott formulas are defined as

$$
\begin{aligned}
\varphi_{0}^{M, \vec{a}}(\vec{x}) & =\bigwedge\{\psi(\vec{x}) \mid \psi \text { quantifier free, } M \models \psi(\vec{a})\} \\
\varphi_{\lambda}^{M, \vec{a}}(\vec{x}) & =\bigwedge_{\beta<\lambda} \varphi_{\beta}^{M, \vec{a}}(\vec{x}), \lambda \text { limit, } \\
\varphi_{\alpha+1}^{M, \vec{a}}(\vec{x}) & =\varphi_{\alpha}^{M, \vec{a}}(\vec{x}) \wedge \bigwedge_{b \in|M|} \exists y \varphi_{\alpha}^{M, \vec{a}, b}(\vec{x}, y) \wedge \forall y \bigvee_{b \in|M|} \varphi_{\alpha}^{M, \vec{a}, b}(\vec{x}, y) .
\end{aligned}
$$

Thus $M \equiv_{\alpha} N$ if and only if $\varphi_{\alpha}^{M, \varnothing}=\varphi_{\alpha}^{N, \varnothing}$.

Another well-known method to detect $\alpha$-equivalence is by playing some twoperson games. Given $\mathcal{L}$-models $M$ and $N$, the Ehrenfeucht-Fraisse game (EF game, for short), denoted $\mathrm{EF}_{\alpha}(M, N)$, is played as follows: Player I starts by playing an ordinal $\alpha_{0}<\alpha$ and an element $x_{0}$ of either model $M$ or $N$; Player II responds by playing an element $y_{0}$ of the opposite model; then in each successive round Player I plays a smaller ordinal and an element of either model, and Player II responds by playing an element of the opposite model; and so on. The play of the game finishes after the round when Player I has played the ordinal 0. The following diagram shows a play of this game.

$$
\begin{aligned}
& \text { I } \alpha_{0}<\alpha, x_{0} \quad \alpha_{1}<\alpha_{0}, x_{1} \quad \ldots \quad \alpha_{n}=0<\alpha_{n-1}, x_{n} \\
& \begin{array}{llll}
y_{0} & y_{1} & \cdots & y_{n}
\end{array}
\end{aligned}
$$

The rule of the game is that $\forall i \leq n\left(x_{i} \in|M| \leftrightarrow y_{i} \in|N|\right)$. Let $\vec{a} \in|M|^{n+1}$, $\vec{b} \in|N|^{n+1}$ be the tuples produced by the demonstrated play. Then Player II wins the play if $(M, \vec{a}) \equiv_{0}(N, \vec{b})$. It is easy to see that $M \equiv_{\alpha} N$ if and only if Player II has a winning strategy in the game $\mathrm{EF}_{\alpha}(M, N)$. 
Now fix an $\mathcal{L}$-model $M$. We recall the definition of Scott rank. In fact, there have been two slightly different definitions in the literature. We give both of them and make some comments on their similarity and difference. For $\vec{a} \in|M|^{n}$, let $\operatorname{sr}_{M}(\vec{a})$ be the least $\alpha<\omega_{1}$ such that for any $\vec{b} \in|M|^{n},(M, \vec{a}) \equiv_{\alpha}(M, \vec{b})$ implies that $(M, \vec{a}) \cong(M, \vec{b})$. Then the two versions of the Scott rank of $M$, respectively denoted $\operatorname{sr}(M)$ and $\operatorname{SR}(M)$, are defined as

$$
\operatorname{sr}(M)=\sup \left\{\left.\operatorname{sr}_{M}(\vec{a})|\vec{a} \in| M\right|^{n}, n>0\right\}
$$

and

$$
\operatorname{SR}(M)=\sup \left\{\operatorname{sr}_{M}(\vec{a})+\left.1|\vec{a} \in| M\right|^{n}, n>0\right\} .
$$

It is easy to see that $\operatorname{sr}(M) \leq \operatorname{SR}(M) \leq \operatorname{sr}(M)+1$. The main difference is that $\operatorname{sr}(M) \leq \omega_{1}^{\mathrm{CK}(M)}$ whereas $\mathrm{SR}(M) \leq \omega_{1}^{\mathrm{CK}(M)}+1$. The difference is significant for some of the studies of countable models (cf. [9] and [8]). In general, SR $(M)$ represents the complexity of $M$ more accurately, while in comparison $\operatorname{sr}(M)$ provides a cruder estimate. However, in view of Sacks's Theorem 1.7, the Borelness of the isomorphism relation is related to Scott ranks in the same manner no matter which definition is employed. For our purposes it suffices to work with $\operatorname{sr}(M)$, following [1] and [13].

We also recall that the canonical Scott sentence of $M$ is defined as

$$
\varphi^{M}=\varphi_{\operatorname{sr}(M)}^{M, \varnothing} \wedge \bigwedge_{\vec{a} \in|M|^{n}}(\exists \vec{x})\left(\varphi_{\operatorname{sr}(M)}^{M, \vec{a}}(\vec{x}) \rightarrow \varphi_{\operatorname{sr}(M)+1}^{M, \vec{a}}(\vec{x})\right) .
$$

The remarkable property of $\varphi^{M}$ is that for any countable $\mathcal{L}$-model $N, N \models \varphi^{M}$ if and only if $N \cong M$.

Note that the quantifier rank of $\varphi^{M}$ goes a little beyond the Scott rank:

$$
\operatorname{qr}\left(\varphi^{M}\right)=\operatorname{sr}(M)+\omega=\operatorname{SR}(M)+\omega .
$$

\section{Variations of the Scott Rank}

3.1 The game rank The Scott analysis yields an ordinal $\alpha \leq \operatorname{sr}(M)+\omega$ for a countable $\mathcal{L}$-model $M$ so that whenever $N$ is a countable $\mathcal{L}$-model with $N \equiv_{\alpha} M$, $N \cong M$. That is to say, whenever Player II has a winning strategy in the game $\mathrm{EF}_{\alpha}(M, N), M$ and $N$ are indeed isomorphic. We thus call the least such ordinal the game rank of $M$ and denote it by $\operatorname{gr}(M)$. We know that $\operatorname{gr}(M) \leq \operatorname{sr}(M)+\omega$.

It turns out that $\operatorname{sr}(M) \leq \operatorname{gr}(M)+\omega$. I learned the following argument from Hjorth, who attributed it to earlier researchers in the field. It seems to be a folklore result which has been rediscovered a number of times, but I did not find an explicit reference.

Proposition 3.1 For any countable model $M, \operatorname{sr}(M) \leq \operatorname{gr}(M)+\omega$.

Proof Let $\alpha=\operatorname{gr}(M)$. Consider the smallest fragment $F$ in $\mathcal{L}_{\omega_{1} \omega}$ containing all Scott formulas $\varphi_{\alpha+k}^{M, \varnothing}$ for $k \in \omega$. Note that for each tuple $\vec{a} \in|M|^{n}$ and $k \in \omega$, the Scott formula $\varphi_{\alpha+k}^{M, \vec{a}}(\vec{x})$ is in $F$ since it is a subformula of $\varphi_{\alpha+k+n}^{M, \varnothing}$. Now let $T$ be the $F$-theory of $M$. In particular, $\varphi_{\alpha}^{M, \varnothing} \in T$. It follows that $M$ is the unique countable model of $T$ and that there are only countably many complete $F$-types for the theory $T$. This implies that an $F$-atomic model of $T$ exists, and therefore $M$ is an $F$-atomic model. For $\vec{a} \in|M|^{n}$ we let $\operatorname{tp}_{F}(\vec{a})$ denote the $F$-type of $\vec{a}$ in $M$. Then $\operatorname{tp}_{F}(\vec{a})$ is principal and there exists a single formula $\psi(\vec{x})$ so that every formula in 
$\operatorname{tp}_{F}(\vec{a})$ is implied by $\psi(\vec{x})$. By our construction $\operatorname{qr}(\psi)<\alpha+\omega$. It follows that for every $\vec{a} \in|M|^{n}$, every $F$-type over $(M, \vec{a})$ is also principal. Thus $(M, \vec{a})$ is an $F$-atomic model of its theory. Now if $(M, \vec{a}) \equiv_{\alpha+k}(M, \vec{b})$, a standard back-andforth argument yields an automorphism $\pi$ of $M$ so that $\pi(\vec{a})=\vec{b}$. This shows that $\operatorname{sr}_{M}(\vec{a})<\alpha+\omega$ for all $\vec{a} \in|M|^{n}$, and hence $\operatorname{sr}(M) \leq \alpha+\omega$.

The game rank is thus a complexity measure for countable models much like the Scott rank. However, we do not have any example of $M$ with $\operatorname{gr}(M)<\operatorname{sr}(M)$. The game rank puts more emphasis on the existence of models: if $\alpha<\operatorname{gr}(M)$ then there exists a model $N$ with $N \equiv_{\alpha} M$ but $N \not M$.

We now turn to other variations of the Scott rank. These are complexity measures related to submodels of the model. First we note that a model of low Scott rank can have submodels of arbitrarily high Scott rank. The countable dense linear order without end points is such an example. Thus it does not make sense to consider arbitrary submodels in general. However, for special classes of models there are distinctive kinds of submodels which cannot have arbitrary Scott ranks. And, in fact, for the classes we consider there is usually a natural type of submodels which we can regard as the "basic building blocks" of our models. Naturally we wonder about their Scott ranks and the connection with the Scott ranks of the ambient model. From now on we will descend to the concrete classes of linear orders and mono-unary algebras. Since the language is rather simple and standard, we will not make any notational difference between a model $M$ and its domain $|M|$.

3.2 Linear orders In linear orders the natural building blocks are intervals, or more generally, segments. Let $(M,<)$ be a linear order and $a<b \in M$. Define

$$
[a, b]^{M}=\{c \in M \mid a \leq c \leq b\} .
$$

It is easily verified that

$$
\operatorname{sr}\left([a, b]^{M}\right) \leq \operatorname{sr}(M) .
$$

Further, we can define the interval rank of $M$ by

$$
\operatorname{ir}(M)=\sup \left\{\operatorname{sr}\left([a, b]^{M}\right) \mid a<b \in M\right\} .
$$

We see that $\operatorname{ir}(M) \leq \operatorname{sr}(M)$. In fact, the interval rank was first defined by Steel in [13]. He also conjectured that the following is true.

Question 3.2 (Steel [13]) $\quad I s \operatorname{sr}(M) \leq(\operatorname{ir}(M)+\omega) \cdot 3$ ?

Steel proved the conjecture in the case there are only countably many nonisomorphic models that are $(\operatorname{ir}(M)+\omega)$-equivalent to $M$. It was also proved in [4] under a weaker hypothesis $E_{0} \not_{B} \cong \uparrow \operatorname{Mod}\left(\equiv_{\mathrm{ir}(M)+\omega} M\right)$. The general case is open. Note that the exact formula is not the point of focus here. As long as the Scott rank of $M$ is bounded by applying a "computable" function on the interval rank of $M$, proofs of various earlier theorems can be simplified and possibly strong dichotomy theorems can be proved.

Another intriguing question is about the game rank of an interval versus the game rank of the whole model.

Question 3.3 If $(M,<)$ is a countable linear order and $a<b \in M$, is

$$
\operatorname{gr}\left([a, b]^{M}\right) \leq \operatorname{gr}(M) ?
$$


Another type of submodel of a linear order is a segment. Let $(M,<)$ be a linear order. A subset $S \subseteq M$ is a segment if for all $a<b<c \in M$, if $a, c \in S$ then $b \in S$. It is trivial that $\operatorname{ir}(S) \leq \operatorname{ir}(M)$. However, we do not know either $\operatorname{sr}(S) \leq \operatorname{sr}(M)$ or $\operatorname{gr}(S) \leq \operatorname{gr}(M)$.

We speculate that the technique needed to resolve all these questions about various complexity ranks of linear orders can be used to prove stronger dichotomy theorems about the isomorphism of linear orders.

3.3 Mono-unary algebras Finally we present some positive results for a class of models for which we have the best knowledge about the isomorphism relation. These are the models in the theory of one unary function. For our purposes we will consider these models in an expanded language $\mathcal{L}$ and call $\mathcal{L}$-models mono-unary algebras.

To define the language, consider a model $\mathbb{A}=(A, f)$ in the language of one unary function. First of all, there is the binary relation $R$ so that, for $x, y \in A$,

$$
R(x, y) \Longleftrightarrow f(x)=y \text {. }
$$

Then we define

$$
x<y \Longleftrightarrow \exists n>0\left(f^{n}(y)=x\right) \wedge \forall m>0\left(f^{m}(x) \neq y\right) .
$$

Finally, for each $l \in \mathbb{Z}$, we define a binary relation $D_{l}$ by

$$
D_{l}(x, y) \Longleftrightarrow \exists n>|l|\left(f^{n}(x)=f^{n+l}(y)\right) \text {. }
$$

We define the relative length of $y$ with respect to $x$, or the length of $y$ relative to $x$, denoted by $\operatorname{lh}_{x}(y)$, to be $l$ if $D_{l}(x, y)$ holds.

Our formal language will be $\mathcal{L}=\{R,<\} \cup\left\{D_{l} \mid l \in \mathbb{Z}\right\}$. There is a sentence $\sigma \in \mathcal{L}_{\omega_{1} \omega}$ axiomatizing the intended class of models in the theory of one unary function. We call the models of $\sigma$ mono-unary algebras. Informally, when we address a mono-unary algebra we will still refer to a function $f$, even if it is not in our formal language.

It is natural to consider the subtrees the basic building blocks for a mono-unary algebra. For our purposes a tree $T$ will mean a partial order $<$ with a least element $r$ (i.e., the root) so that for any $t \in T$, the set $\{s \in T \mid s<t\}$ is finite and linearly ordered by $<$. We can define

$$
R(s, t) \Longleftrightarrow t<s \wedge \neg \exists u(t<u<s) .
$$

Then $R$ is the graph of a function $f$ (in fact a partial one, since $f(r)$ is undefined) and from it we can define the relations $D_{l}, l \in \mathbb{Z}$, as before. In this sense we regard trees as formally mono-unary algebras. For $t \in T$ we will speak of the length of $t$, denoted by $\operatorname{lh}_{T}(t)$, which is the cardinality of the set $\{s \in T \mid s<t\}$. In fact, $\mathrm{lh}_{T}(t)=\mathrm{lh}_{r}(t)$. When there is no danger of confusion we will drop the subscripts and simply denote it by $\operatorname{lh}(t)$. For each $x \in A$ let

$$
\mathbb{A}(x)=\{y \in A \mid x \leq y\} .
$$

Then each $\mathbb{A}(x)$ is a tree.

A mono-unary algebra $\mathbb{A}$ is connected if $\forall x, y \in A \exists z \in A(x \leq z \wedge y \leq z)$. A general mono-unary algebra can be decomposed into connected components, that is, connected subalgebras. There are, however, two different types of connected monounary algebras. If $\mathbb{A}$ is connected, we call it type $I$ if $\exists x \in A \exists n>0\left(f^{n}(x)=x\right)$, and type II otherwise. In graph theoretic terms, a type I algebra is essentially a tree with possibly a cycle at the root. For a type I algebra the study of subtrees is 
essentially equivalent to the study of the whole algebra. Thus our main focus will be type II algebras.

We can now define the tree rank of $A$ to be

$$
\operatorname{tr}(\mathbb{A})=\sup \{\operatorname{sr}(\mathbb{A}(x)) \mid x \in \mathbb{A}\} .
$$

We have the following facts showing that all the ranks considered are close to each other. Note also that the last inequality below gives a positive answer to the analog of Steel's question.

Proposition 3.4 Let $\mathbb{A}$ be a mono-unary algebra. Then the following hold:

(i) $\operatorname{tr}(\mathbb{A}) \leq \operatorname{sr}(\mathbb{A})$;

(ii) $\operatorname{tr}(\mathbb{A}) \leq \operatorname{gr}(\mathbb{A})+\omega$;

(iii) $\operatorname{gr}(\mathbb{A}) \leq \omega+\sup \{\operatorname{gr}(\mathbb{A}(x)) \mid x \in \mathbb{A}\}+\omega$;

(iv) $\operatorname{gr}(\mathbb{A}) \leq \omega+\operatorname{tr}(\mathbb{A})+\omega \cdot 2$;

(v) $\operatorname{sr}(\mathbb{A}) \leq \omega+\operatorname{tr}(\mathbb{A})+\omega \cdot 3$.

Proof (i) is easy to see and (ii) follows from Proposition 3.1. For (iii) we let $\alpha=\omega+\sup \{\operatorname{gr}(\mathbb{A}(x)) \mid x \in \mathbb{A}\}$ and assume toward a contradiction that $\alpha+\omega<\operatorname{gr}(\mathbb{A})$. Then there exists an algebra $\mathbb{B}$ such that $\mathbb{A} \equiv_{\alpha+\omega} \mathbb{B}$ but $\mathbb{A} ¥ \mathbb{B}$. For each connected component $C$ of $\mathbb{A}$ let $N_{\mathbb{A}}(C)$ be the the number of distinct connected components of $\mathbb{A}$ isomorphic to $C$ and let $N_{\mathbb{B}}(C)$ be the number of distinct connected components of $\mathbb{B}$ isomorphic to $C$. We may assume without loss of generality that there is a connected component $C$ of $\mathbb{A}$ such that $N_{\mathbb{A}}(C)>N_{\mathbb{B}}(C)$. Let $n=N_{\mathbb{B}}(C)$. Then in a play of the game $\mathrm{EF}_{\alpha+\omega}(\mathbb{A}, \mathbb{B})$ let Player I play for at least $n+1$ rounds with all ordinals $>\alpha$ and elements from at least $n+1$ distinct connected components all of which are isomorphic to $C$. If Player II follows her winning strategy then she must play elements from distinct connected components of $\mathbb{B}$. To see this, assume her responses contain two elements $b_{1}$ and $b_{2}$ from the same connected component. Suppose $a_{1}$ and $a_{2}$ are elements of $\mathbb{A}$ Player I played that are correspondent to $b_{1}$ and $b_{2}$, respectively. Then since $\alpha \geq \omega$ Player I can win the rest of the play by playing finitely many witnesses of connectedness of $b_{1}$ and $b_{2}$. Since $a_{1}$ and $a_{2}$ are not in the same connected component there is no way for Player II to appropriately respond so as not to lose. This contradicts our assumption that Player II was following her winning strategy. Thus among the $n+1$ elements Player II played we can identify an element and its connected component $D$ of $\mathbb{B}$ so that $C \not D$ but $C \equiv_{\alpha+1} D$. Without loss of generality assume Player I played the ordinal $\alpha+1$ and $a \in C$ in his $(n+1)$ th move and Player II responded to this move by playing $b \in D$. Since $C \not D$, there is some $m \in \omega$ such that $C\left(f^{m}(a)\right) \nRightarrow C\left(f^{m}(b)\right)$. Continue to play one more round of the game by letting Player I play $\alpha$ and $f^{m}(a)$. We note that if Player II follows her winning strategy she must respond by playing $f^{m}(b)$, otherwise Player I can win the play by continuing to play all elements of the set $\left\{f^{i}(a) \mid 0<i<n\right\}$. Let $c=f^{m}(a)$ and $d=f^{m}(b)$. Then we see that $C(c) \nsucceq D(d)$ and $C(c) \equiv_{\alpha} D(d)$. This shows that $\mathbb{A}(c)=C(c)$ has game rank $>\alpha$, contradicting our assumption. We have thus proved (iii). Now (iv) and (v) follow from (iii) and Proposition 3.1.

Our main theorem of this article is about the game rank of subtrees versus the game rank of the whole algebra.

Theorem 3.5 For any $x \in \mathbb{A}, \operatorname{gr}(\mathbb{A}(x)) \leq \omega+\operatorname{gr}(\mathbb{A})$. 
The proof is probably more significant than the statement of the theorem since it unravels a detailed local analysis of the isomorphism of mono-unary algebras in terms of their subtrees. In contrast, our Question 3.3 is a confession that we lack such an analysis for linear orders. The rest of the paper is devoted to a proof of Theorem 3.5.

\section{Preliminaries for the Proof of the Main Theorem}

We first reduce the problem to the special case where the algebra is connected and type II.

Lemma 4.1 Let $\mathbb{A}$ be a mono-unary algebra and $C$ be a connected component of A. Then $\operatorname{gr}(C) \leq \omega+\operatorname{gr}(\mathbb{A})$.

Proof Let $\alpha=\omega+\operatorname{gr}(\mathbb{A})$. Assume toward a contradiction that $\alpha<\operatorname{gr}(C)$. Then there exists an algebra $D$ with $C \equiv_{\alpha} D$ but $D \not C$. Since $\alpha \geq \omega$ we see that $D$ is connected. Let $n \leq \omega$ be the number of all distinct connected components of $\mathbb{A}$ $\alpha$-equivalent to $C$. Let $C_{0}=C, C_{1}, \ldots, C_{k}, \ldots, k<n$, enumerate these connected components. Obtain an algebra $\mathbb{B}_{1}$ from $\mathbb{A}$ by replacing each $C_{k}, k<n$, by a copy of $C$. Clearly, $\mathbb{B}_{1} \equiv_{\alpha}$ A. Similarly, obtain another algebra $\mathbb{B}_{2}$ from $\mathbb{A}$ by replacing each $C_{k}, k<n$, by a copy of $D$. Then again $\mathbb{B}_{2} \equiv_{\alpha} A$. Now note that $\mathbb{B}_{1} \not \mathbb{B}_{2}$, since $C$ is not isomorphic to any connected component of $\mathbb{B}_{2}$. Thus at least one of $\mathbb{B}_{1}$ and $\mathbb{B}_{2}$ is not isomorphic to $\mathbb{A}$. This shows that $\alpha<\operatorname{gr}(\mathbb{A})$, contradiction.

In view of the above lemma it suffices to prove the theorem for connected monounary algebras. Since type I algebras are essentially trees, the theorem is trivial for them. From now on we fix a type II connected mono-unary algebra A.

We need to digress to some notation and constructions to be repeatedly used later in the proof. Let $T$ be a (rooted) tree ordered by <. Two elements $s, t \in T$ are incomparable if neither $s \leq t$ nor $t \leq s$, in which case we write $s \perp t$. An antichain in $T$ is a subset $X \subseteq T$ such that $s \perp t$ for all distinct $s, t \in X$. Note that these definitions make sense for any partial order, in particular, any mono-unary algebra.

We write $T \hookrightarrow \mathbb{A}$ if there is $x \in \mathbb{A}$ such that $T \cong \mathbb{A}(x)$. Let $S$ also be a tree (possibly the same as $T$ ). Then we also write $T \hookrightarrow S$ if there is $s \in S$ with $\operatorname{lh}(s)>0$ such that $T \cong S(s)$.

Let $T$ be a tree, $\mathbb{B}$ be a mono-unary algebra, and $x \in \mathbb{B}$. We denote by $\Upsilon(\mathbb{B}, x, T)$ the algebra obtained from $\mathbb{B}$ by replacing $\mathbb{B}(x)$ by $T$. Formally, the domain of $\Upsilon(\mathbb{B}, x, T)$ is the disjoint union of $\mathbb{B}-\mathbb{B}(x)$ with $T$, and the partial order $<$ is defined by

$y<z \Longleftrightarrow(y, z \in \mathbb{B} \wedge y<z) \vee(y, z \in T \wedge y<z) \vee(y \in \mathbb{B} \wedge y<x \wedge z \in T)$.

Thus formally $x$ is no longer an element of $\Upsilon(\mathbb{B}, x, T)$. However, for notational convenience later we will regard that the root of $T$ be identified with $x$, and we will call it $x$ in $\Upsilon(\mathbb{B}, x, T)$. This convention will apply to all other similar constructions below.

Let $\mathbb{B}$ be a mono-unary algebra, $n \leq \omega$, and $x_{0}, x_{1}, \ldots, x_{k}, \ldots, k<n$, be a sequence of elements in $\mathbb{B}$ so that the set $X=\left\{x_{k} \mid k<n\right\}$ is an antichain in $\mathbb{B}$. Let $T_{0}, T_{1}, \ldots, T_{k}, \ldots, k<n$, be a sequence of trees. We denote by $\Upsilon(\mathbb{B}, \vec{x}, \vec{T})$ the algebra obtained from $\mathbb{B}$ by simultaneously replacing, for all $k<n, \mathbb{B}\left(x_{k}\right)$ by $T_{k}$, respectively. A formal definition similar to the above can be given, but we omit it 
here. Just note that the algebra is well defined only when $X$ is an antichain. In case all the $T_{k}$ s are the same tree $T$, we also write $\Upsilon(\mathbb{B}, X, T)$ for $\Upsilon(\mathbb{B}, \vec{x}, \vec{T})$.

One further construction will be especially important, so we treat it separately here. Let $T$ and $S$ be trees and $x_{0} \in S$. Define a sequence $S_{n}, n \in \omega$, of trees by induction on $n$. Let $S_{0}=S$ and $S_{1}=\Upsilon\left(S, x_{0}, T\right)$. For $n>0$, let $S_{n+1}=\Upsilon\left(S, x_{0}, S_{n}\right)$. Intuitively, $S_{n+1}$ contains $n$ copies of $S-S\left(x_{0}\right)$, one on top of another, with a copy of $T$ at the bottom (see Figure 1). We first note a simple fact.

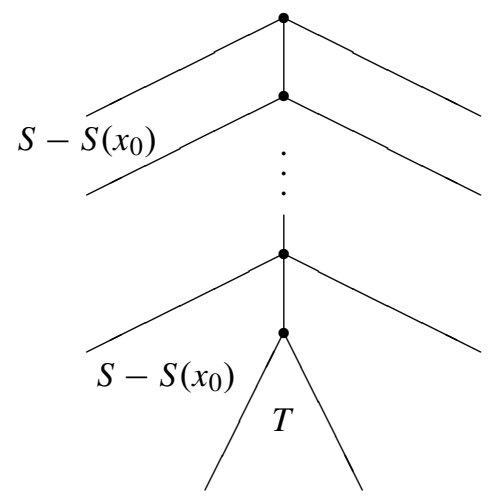

Figure 1 The tree $S_{n}$.

Lemma 4.2 If $S \equiv_{\alpha} S\left(x_{0}\right) \equiv_{\alpha} T$, then for all $n \in \omega, S_{n} \equiv_{\alpha} T$.

Proof Let $\tau$ be a winning strategy for Player II in the game $\mathrm{EF}_{\alpha}\left(S\left(x_{0}\right), T\right)$. We first describe a winning strategy $\tau_{0,1}$ for Player II in the game $\mathrm{EF}_{\alpha}\left(S_{0}, S_{1}\right)$. Suppose before round $k$ ordinals $\alpha_{0}>\alpha_{1}>\cdots>\alpha_{k-1}$ have been played and elements $y_{0}, \ldots, y_{k-1} \in S_{0}$ and $z_{0}, \ldots, z_{k-1} \in S_{1}$ have been played. Suppose Player I played $\alpha_{k}<\alpha_{k-1}$ and $u \in S_{0} \cup S_{1}$ in round $k$. First consider the case $u \in S_{0}$. If $u \notin S\left(x_{0}\right)$ then we let Player II respond by playing $u$. If $u \in S\left(x_{0}\right)$ then for $0 \leq i<k$ we let

$$
u_{i}= \begin{cases}y_{i}, & \text { if } y_{i} \in S\left(x_{0}\right), \\ x_{0}, & \text { otherwise }\end{cases}
$$

and

$$
v_{i}= \begin{cases}z_{i}, & \text { if } z_{i} \in S\left(x_{0}\right), \\ x_{0}, & \text { otherwise. }\end{cases}
$$

Now by inductive hypothesis the position with ordinals $\alpha_{0}, \ldots, \alpha_{k-1}, \alpha_{k}$ and elements $u_{0}, \ldots, u_{k-1}, u \in S_{0}$ and $v_{0}, \ldots, v_{k-1}$ played is compatible with the strategy $\tau$. We apply $\tau$ to this position and let Player II play the resulting element $v \in T=S_{1}\left(x_{0}\right)$. For the case $u \in S_{1}$ a similar definition can be given to yield an element $v \in S_{0}$. This defines a strategy $\tau_{0,1}$ for Player II in $\operatorname{EF}_{\alpha}\left(S_{0}, S_{1}\right)$, which is essentially a natural combination of the copying strategy with the strategy $\tau$. It is easy to see that $\tau_{0,1}$ is winning.

Now since $S_{1} \equiv_{\alpha} S_{1}\left(x_{0}\right)=T \equiv_{\alpha} S_{1}$, applying the same argument as above for the next step of the construction yields a winning strategy $\tau_{1,2}$ for Player II in the game $\mathrm{EF}_{\alpha}\left(S_{1}, S_{2}\right)$. By induction we get that $S_{n} \equiv_{\alpha} S_{n+1}$ for all $n \in \omega$. 
In general suppose $X$ is an antichain in $\mathbb{B}$ and for all $x \in X, \mathbb{B}(x) \equiv_{\alpha} T$, then $\mathbb{B} \equiv_{\alpha} \Upsilon(\mathbb{B}, X, T)$. This can be seen by combining the copying strategy and the winning strategies in the games $\mathrm{EF}_{\alpha}(\mathbb{B}(x), T)$ in a similar manner to the above proof.

For the sequence of trees $S_{n}$, it is important for us to know more details about these strategies involved in the proof. If $\tau$ is a strategy for Player II in the game $\mathrm{EF}_{\alpha}(S, T)$ for some trees $S$ and $T$, then we call $\tau$ regular if the following condition holds: for any play of the game $\mathrm{EF}_{\alpha}(S, T)$ compatible with $\tau$, if $s \in S$ and $t \in T$ are played in a single round, then $\ln (s)=\operatorname{lh}(t)$.

Lemma 4.3 If $S \equiv_{\alpha} T$ where $\alpha \geq \omega$, then there exists a regular winning strategy $\tau$ for Player II in the game $\mathrm{EF}_{\alpha}(S, T)$.

Proof Let $\tau_{0}$ be a winning strategy for Player II in the game $\mathrm{EF}_{\alpha}(S, T)$. We describe a regular winning strategy $\tau$ for Player II. Suppose Player I's first move consists of $\alpha_{0}<\alpha$ and $u \in S \cap T$. If $\alpha_{0} \geq \omega$, then from now on we let Player II follow strategy $\tau_{0}$. We check that in each round of the play the lengths of the elements played are the same. First, let $s_{0} \in S$ and $t_{0} \in T$ be played in the first round. Then $\operatorname{lh}\left(s_{0}\right)=\operatorname{lh}\left(t_{0}\right)$. Otherwise, assume without loss of generality, that $\operatorname{lh}\left(s_{0}\right)<\operatorname{lh}\left(t_{0}\right)=n$; then Player I can win by playing $n$ more rounds in which he plays all elements of the set $\left\{t \mid t_{0}<t\right\}$, contradicting that $\tau_{0}$ is winning. Now suppose $s \in S$ and $t \in T$ be elements played in another round; then we must have that $D_{l}\left(s_{0}, s\right)$ if and only if $D_{l}\left(t_{0}, t\right)$ for all $l \in \mathbb{Z}$, which implies that $\mathrm{lh}_{s_{0}}(s)=\mathrm{lh}_{t_{0}}(t)$. Then $\operatorname{lh}(s)=\operatorname{lh}_{s_{0}}(s)+\operatorname{lh}\left(s_{0}\right)=\operatorname{lh}_{t_{0}}(t)+\operatorname{lh}\left(t_{0}\right)=\operatorname{lh}(t)$.

If $\alpha_{0}<\omega$ and $u \in S \cap T$ with $\operatorname{lh}(u)=n$, then let Player II apply $\tau_{0}$ to the position with ordinal $\omega$ and $u$ and follow $\tau_{0}$ from then on. By the argument above the responses will have the same lengths as Player I's moves in the same round. This defines a regular strategy $\tau$ for Player II. It is easy to see that $\tau$ is winning.

Now back to the proof of Lemma 4.2. Suppose $\alpha \geq \omega$ and let $\tau$ be a regular winning strategy for Player II in the game $\mathrm{EF}_{\alpha}\left(S\left(x_{0}\right), T\right)$. Let $\operatorname{lh}\left(x_{0}\right)=l$. For each $n \in \omega$, let $\tau_{n, n+1}$ be the winning strategy constructed for Player II in the game $\mathrm{EF}_{\alpha}\left(S_{n}, S_{n+1}\right)$. It takes an easy induction to see that every $\tau_{n, n+1}$ is regular. In particular, for any element $t$ in $S_{n}$ (or $S_{n+1}$ ) played by Player I in this game, if $\operatorname{lh}(t) \leq n l$ then Player II's response following $\tau$ is essentially a copy of $t$ in $S_{n+1}$ (or $S_{n}$, respectively). Furthermore, for any $m, n \in \omega$ with $m<n$, a winning strategy $\tau_{m, n}$ for Player II in the game $\mathrm{EF}_{\alpha}\left(S_{m}, S_{n}\right)$ can be obtained by naturally composing the strategies $\tau_{m, m+1}, \ldots, \tau_{n-1, n}$. It follows that $\tau_{m, n}$ is regular too. If $t$ is played by Player I with $\mathrm{lh}(t) \leq m l$, then Player II's response following $\tau_{m, n}$ is essentially a copy of $t$.

We have just noted that the first $n l$ levels of $S_{n}$ agree with those of $S_{n+1}$, and thus we can identify them and consider the first $n l$ levels of $S_{n}$ a subset of $S_{n+1}$. This allows us to construct a direct limit $S_{\omega}$ of the sequence of models $S_{n}, n \in \omega$. Specifically, let $S_{\omega}$ be the union of first $n l$ levels of $S_{n}$ for all $n \in \omega$. For any $s, t \in S_{\omega}$, if $\operatorname{lh}(s), \operatorname{lh}(t)<n l$, then both $s, t \in S_{n}$ and $s<t$ in $S_{\omega}$ exactly when $s<t$ in $S_{n}$. The property we want from $S_{\omega}$ is its $\alpha$-equivalence to $T$.

Lemma 4.4 If $S \equiv_{\alpha} S\left(x_{0}\right) \equiv_{\alpha} T$ where $\alpha \geq \omega$, then $S_{\omega} \equiv_{\alpha} T$.

Proof Let $\tau$ be a regular winning strategy for Player II in the game $\mathrm{EF}_{\alpha}\left(S\left(x_{0}\right), T\right)$. Let $\tau_{m, n}, m<n$, be the regular winning strategies constructed above. We describe a winning strategy $\tau_{0, \omega}$ for Player II in the game $\operatorname{EF}_{\alpha}\left(S_{0}, S_{\omega}\right)$. For this let Player I's 
first move consist of $\alpha_{0}$ and $u \in S_{0} \cup S_{\omega}$. First assume $u \in S_{0}$. Then consider all games $\mathrm{EF}_{\alpha}\left(S_{0}, S_{n}\right)$ and apply the strategies $\tau_{0, n}$ to obtain a response in each of $S_{n}$. As noted above, for any $n$ such that $\operatorname{lh}(u) \leq n l$, the response in $S_{n}$ is essentially the same as that in $S_{m}$ for all $m \geq n$, which is also an element of $S_{\omega}$. We let Player II play this element as her response in $S_{\omega}$. Alternatively, suppose $u \in S_{\omega}$. Then again for all $n$ such that $\operatorname{lh}(u) \leq n l$ we have a copy of $u$ in each $S_{n}$. Note that for all such $n$ we obtain a unique result by applying the strategy $\tau_{0, n}$. Let Player II play this unique element as her response. The other rounds of the game are played in the same fashion. This describes a regular strategy for Player II in the game $\mathrm{EF}_{\alpha}\left(S_{0}, S_{\omega}\right)$. To see that it is winning, consider an arbitrary play of this game. Suppose that it consists of $\alpha_{0}=\alpha>\cdots>\alpha_{k}=0, u_{0}, \ldots, u_{k} \in S_{0}$ and $v_{0}, \ldots, v_{k} \in S_{\omega}$. Then there is some $n$ such that $\operatorname{lh}\left(u_{i}\right) \leq n l$ for all $i \leq k$. Now the play considered can be regarded as a play in the game $\mathrm{EF}_{\alpha}\left(S_{0}, S_{n}\right)$ and by our construction is compatible with the strategy $\tau_{0, n}$. Thus Player II has won.

\section{Proof of the Main Theorem}

Let $\mathbb{A}$ be a type II connected mono-unary algebra and $x \in \mathbb{A}$. We show that $\operatorname{gr}(\mathbb{A}(x)) \leq \max \{\omega, \operatorname{gr}(\mathbb{A})\}$. For this let $T$ be $\mathbb{A}(x)$ and $\alpha=\max \{\omega, \operatorname{gr}(\mathbb{A})\}$. Assume that $\alpha<\operatorname{gr}(T)$. Then we can fix a tree $S$ with $S \equiv_{\alpha} T$ but $S \not T T$. We will produce an algebra $\mathbb{B}$ so that $\mathbb{B} \equiv_{\alpha} \mathbb{A}$ but $\mathbb{B} \nsucceq \mathbb{A}$. This will be done by considering several cases.

Case $1 T \hookrightarrow S$ and $S \hookrightarrow T$. In this case consider $V=\{y \in \mathbb{A}: \mathbb{A}(y) \cong T\}$. Let $W$ be a maximal antichain in $V$. Let $X=(\mathbb{A}-\bigcup\{\mathbb{A}(y) \mid y \in W\}) \cup W$. Define $\mathbb{B}=\Upsilon(\mathbb{A}, W, S)$. Then $\mathbb{A} \equiv_{\alpha} \mathbb{B}$. To see that $\mathbb{B} \not \mathbb{A}$, we claim that there is no $u \in \mathbb{B}$ with $\mathbb{B}(u) \cong T$. Assume to the contrary that $\mathbb{B}(u) \cong T$. Then, for any $y \in W$, $\mathbb{B}(y) \cong S$, and thus $y \not z u$, since $T \not S$ and $T \hookrightarrow S$. Thus $u \in X$, and in fact $u \in V$. Since $W$ is a maximal antichain in $V$, there must be $y \in W$ with $u<y$. But then $S \cong \mathbb{B}(y) \hookrightarrow \mathbb{B}(u) \cong T$, a contradiction to our case assumption.

Case $2 \quad T \hookrightarrow S$ but $S \hookrightarrow T$. The proof of this case is much longer than that for Case 1. Again let $V=\{y \in \mathbb{A} \mid \mathbb{A}(y) \cong T\}$. We need to consider four subcases.

Subcase 2.1 There is an infinite decreasing chain in $V$. In this case let $y$ be any element on this chain and define $\mathbb{B}=\Upsilon(\mathbb{A}, y, S)$. Then clearly $\mathbb{B} \equiv_{\alpha} \mathbb{A}$. To see $\mathbb{B} \nsucceq \mathbb{A}$, we claim that in $\mathbb{B}$ there is no infinite decreasing chain of elements $z$ with $\mathbb{B}(z) \cong T$. Assume $z_{1} \geq z_{2} \geq \cdots$ is such a decreasing chain. Since $\mathbb{B}$ is connected, there is some $i$ with $z_{i} \leq y$. But then $S \cong \mathbb{B}(y) \hookrightarrow \mathbb{B}\left(z_{i}\right) \cong T$, a contradiction to our case assumption.

In the remaining three subcases we assume that there is no infinite decreasing chain in $V$; that is, $V$ is well-founded. Let $W$ be the set of minimal elements of $V$. Then $W$ is a maximal antichain of $V$. We need to construct more trees to be substituted in the algebra as follows. Define $U_{0}=S$. Since $T \hookrightarrow S$, we let $l_{0}$ be the least $l$ such that for some $u \in U_{0}$ with $\operatorname{lh}(u)=l, U_{0}(u) \cong T$. Now in $U_{0}$ let $X_{0}=\left\{u \in U_{0} \mid \operatorname{lh}(u)=l_{0}, U_{0}(u) \cong T\right\}$. Define $U_{1}=\Upsilon\left(U_{0}, X_{0}, S\right)$. Then $U_{1} \equiv_{\alpha} U_{0}$ similar to Lemma $4.2, U_{1} \nsucceq T$ since $S \hookrightarrow \hookrightarrow T$, and $U_{1} \not U_{0}$ since for no element $u$ of length $l_{0}$ in $U_{1}$ we have $U_{1}(u) \cong T$. Now let $l_{1}$ be the least $l$ such that for some $u \in U_{1}$ with $\operatorname{lh}(u)=l, U_{1}(u) \cong T$. Then $l_{1}>l_{0}$. Let 
$X_{1}=\left\{u \in U_{1} \mid \operatorname{lh}(u)=l_{1}, U_{1}(u) \cong T\right\}$. Define $U_{2}=\Upsilon\left(U_{1}, X_{1}, S\right)$. Continuing in this fashion we can obtain $U_{n}$ for all $n \in \omega$ so that

(a) $U_{n} \equiv_{\alpha} U_{0}=S$ for all $n$;

(b) $U_{n} \neq U_{m}$ for $n \neq m ; U_{n} ¥ T$;

(c) letting $l_{n}$ be the least $l$ such that for some $u \in U_{n}$ with $\operatorname{lh}(u)=l, U_{n}(u) \cong T$, we have that $l_{n}>l_{m}$ for $n>m$.

We are now ready to continue considering the other subcases of Case 2. Consider the set $W$ defined above and let

$$
L=\inf \left\{\operatorname{lh}_{x}(y) \mid y \in W\right\} .
$$

Then $L \in \mathbb{Z} \cup\{-\infty\}$.

Subcase 2.2 $L=-\infty$. In this case let $W^{\prime}=\left\{y \in W \mid \mathrm{lh}_{x}(y)<0\right\}$. Enumerate $W^{\prime}$ as a sequence $y_{0}, y_{1}, \ldots$, which is denoted by $\vec{y}$. For $i \in \omega$, let $k_{i}=\operatorname{lh}_{x}\left(y_{i}\right)$. For each $i \in \omega$, let $n_{i}$ be the least $n$ such that $l_{n} \geq\left|k_{i}\right|$. Let $\vec{U}$ be the sequence $U_{n_{0}}, U_{n_{1}}, \ldots$ Define $\mathbb{B}=\Upsilon(\mathbb{A}, \vec{y}, \vec{U})$. It is clear that $\mathbb{B} \equiv_{\alpha} \mathbb{A}$. To see that $\mathbb{B} \not \mathbb{A}$, we note from our construction that if $z \in \mathbb{B}$ with $\mathbb{B}(z) \cong T$, then $\mathrm{lh}_{x}(z) \geq 0$.

Subcase 2.3 $L \in \mathbb{Z}$ and $|W|>1$. Fix $y_{0} \in W$ with $\operatorname{lh}_{x}\left(y_{0}\right)=L$. Let $L^{\prime}=\inf \left\{\operatorname{lh}_{x}(y) \mid y \in W, y \neq y_{0}\right\}$. By our assumption $L^{\prime} \geq L$. Now let $W^{\prime \prime}=\left\{y \in W \mid y \neq y_{0}, \operatorname{lh}_{x}(y)=L^{\prime}\right\}$. Define $\mathbb{B}=\Upsilon\left(\mathbb{A}, W^{\prime \prime}, S\right)$. Then $\mathbb{B} \equiv_{\alpha} \mathbb{A}$ but $\mathbb{B} ¥ \mathbb{A}$. This is because $y_{0}$ is the unique element in $\mathbb{B}$ with the smallest relative length (with respect to any element) so that $\mathbb{B}\left(y_{0}\right) \cong T$. By our construction, in $\mathbb{B}$ there is no $y$ with $\operatorname{lh}_{y_{0}}(y)=L^{\prime}-L$ and $\mathbb{B}(y) \cong T$.

Subcase 2.4 $|W|=1$. Let $y_{0}$ be the unique element of $W$. We define a sequence $z_{i} \in \mathbb{A}, i \in \omega$, as follows. Let $z_{0}=f^{i}\left(y_{0}\right)$ where $i \in \omega$ is the smallest such that $\mathbb{A}\left(z_{0}\right) \cong S$. If $z_{0}$ does not exist, then we claim that there is no $u \in \mathbb{A}$ with $\mathbb{A}(u) \cong S$. Assume to the contrary that $u$ is such. If $u \geq y_{0}$ then it contradicts $S \hookrightarrow T$ or $S \neq T$. If $u \perp y_{0}$, then from $T \hookrightarrow S$ it follows that there is a $y \geq u$ with $\mathbb{A}(y) \cong T$; since $y \perp y_{0}$, this contradicts that $|W|=1$. Therefore, if $z_{0}$ does not exist and we define $\mathbb{B}=\Upsilon\left(\mathbb{A}, y_{0}, S\right)$, then $\mathbb{B} \equiv_{\alpha} \mathbb{A}$ but $\mathbb{B} \not \mathbb{A}$ since $S \hookrightarrow \mathbb{B}$ while $S \hookrightarrow \mathbb{A}$.

Thus we assume that $z_{0}$ does exist. Then note that in $S$ there is a largest $x_{0}$ with $S\left(x_{0}\right) \cong T$, since otherwise in $\mathbb{A}$ there would be $y \perp y_{0}$ with $\mathbb{A}(y) \cong T$, contradicting that $W=\left\{y_{0}\right\}$. Let $l_{0}=\operatorname{lh}\left(x_{0}\right)$. Then we must have that $z_{0}=f^{l_{0}}\left(y_{0}\right)$. In fact, if we let $S_{0}=S$, then the construction in Section 4 yields infinitely many trees $S_{1}, S_{2}, \ldots$ so that $S_{n} \equiv_{\alpha} T$ for all $n \in \omega$. Moreover, for each $n>0$, there is a largest $u_{n} \in S_{n}$ with $S_{n}\left(u_{n}\right) \cong T$ and, in fact, $\operatorname{lh}\left(u_{n}\right)=n l_{0}$. Therefore we get that $S_{n} \nsucceq S_{m}$ for distinct $n, m>0$.

Getting back to our definition of the sequence $z_{i}$, we continue to define $z_{i}=f^{i l_{0}}\left(z_{0}\right)=f^{(i+1) l_{0}}\left(y_{0}\right)$ for all $i>0$. If for some $i>0, \mathbb{A}\left(z_{i}\right) \not S_{i}$, then it is easy to see that $S_{i} \hookrightarrow \mathbb{A}$; letting $\mathbb{B}=\Upsilon\left(\mathbb{A}, z_{i-1}, S_{i}\right)$, or equivalently, letting $\mathbb{B}=\Upsilon\left(\mathbb{A}, y_{0}, S\right)$, we have that $\mathbb{B} \equiv_{\alpha} \mathbb{A}$ but $\mathbb{B} ¥ \mathbb{A}$ since $S_{i} \hookrightarrow \mathbb{B}$.

Thus we only need to consider the situation when $\mathbb{A}\left(z_{i}\right) \cong S_{i}$ for all $i \in \omega$. In this situation we use the tree $S_{\omega}$ constructed in Section 4. Define $\mathbb{B}=\Upsilon\left(\mathbb{A}, y_{0}, S_{\omega}\right)$. Since $\alpha \geq \omega$ and therefore $S_{\omega} \equiv_{\alpha} T$, we have that $\mathbb{B} \equiv_{\alpha} \mathbb{A}$. It suffices to show that $\mathbb{B} \not \mathbb{A}$. In fact, if $T \hookrightarrow \mathbb{B}$ then we are done since $T \hookrightarrow \mathbb{A}$. Otherwise, assume $T \hookrightarrow \mathbb{B}$. Note now $\mathbb{B}$ has the property that for any $y \in \mathbb{B}$ there is $y^{\prime} \leq y$ with $\mathbb{B}\left(y^{\prime}\right) \cong S_{\omega}$. So if $T \hookrightarrow \mathbb{B}$ then $T \hookrightarrow S_{\omega}$ and, moreover, by the above property we 
have that

$$
\inf \left\{\operatorname{lh}_{y_{0}}(y) \mid \mathbb{B}(y) \cong T\right\}=-\infty .
$$

In contrast $|W|=1$ and therefore $\inf \left\{\operatorname{lh}_{x}(y) \mid \mathbb{A}(y) \cong T\right\}>-\infty$. Hence, $\mathbb{B} ¥ \mathbb{A}$. This completes our proof for Case 2 .

Case $3 T \hookrightarrow S$ but $S \hookrightarrow T$. Let $\mathbb{A}^{\prime}$ be obtained from $\mathbb{A}$ by substituting a copy of $T$ by $S$. Then switching the roles of $S$ and $T$, we can repeat the argument of Case 2 for $\mathbb{A}^{\prime}$ and obtain $\mathbb{B}^{\prime}$ with $\mathbb{B}^{\prime} \equiv_{\alpha} \mathbb{A}^{\prime}$ and $\mathbb{B}^{\prime} ¥ \mathbb{A}^{\prime}$. Now being nonisomorphic to each other, one of $\mathbb{A}^{\prime}$ and $\mathbb{B}^{\prime}$ must be nonisomorphic to $\mathbb{A}$, and we can let it be $\mathbb{B}$. This finishes the proof for Case 3.

Case $4 \quad T \hookrightarrow S$ and $S \hookrightarrow T$. Before we consider various subcases we construct as many $\alpha$-equivalent nonisomorphic copies of $T$ as possible. For this we define, for any tree $P$, the set $N(P)=\left\{u \in P \mid \operatorname{lh}(u)>0, P(u) \equiv_{\alpha} T\right\}$. Let $M(P)$ be the set of minimal elements of $N(P)$. Then elements of $M(P)$ are pairwise incomparable. Now consider $M(S)$, which is nonempty by our case assumption.

First suppose that $M(S)$ is infinite. Fix $u_{0} \in M(S)$. Let $P_{0}=S$. Consider two trees $P_{1,1}=\Upsilon(S, M(S), T)$ and $P_{1,2}=\Upsilon(S, M(S), S)$. Then in $P_{1,1}$ we have that $P_{1,1}(u) \cong T$ for any $u \in M\left(P_{1,1}\right)$, while in contrast, in $P_{1,2}$ we have that $P_{1,2}(u) \cong S$ for any $u \in M\left(P_{1,2}\right)$. This shows that $P_{1,1} \approx P_{1,2}$, and thus at least one of them is not isomorphic to $P_{0}$. Let $P_{1}$ be $P_{1,1}$ if $P_{1,1} ¥ P_{0}$, and $P_{1,2}$ otherwise. In general, suppose we have found $P_{0}, \ldots, P_{n}(n \geq 1)$ all $\alpha$-equivalent to $T$ but pairwise nonisomorphic. Then, for $0 \leq i \leq n$, let $P_{n+1, i}=\Upsilon\left(S, M(S), P_{i}\right)$, and let $P_{n+1, n+1}$ be obtained from $S$ by substituting $S\left(u_{0}\right)$ by $P_{0}$ and for all $u \in M(S)-\left\{u_{0}\right\}$, $S(u)$ by $P_{1}$. Then it is clear that $P_{n+1,0}, P_{n+1,1}, \ldots, P_{n+1, n}, P_{n+1, n+1}$ are all $\alpha-$ equivalent but pairwise nonisomorphic. It follows that there is $0 \leq i \leq n+1$ such that $P_{n+1, i} ¥ P_{k}$ for all $0 \leq k \leq n$. Let $P_{n+1}$ be $P_{n+1, i}$ where $i$ is the smallest index with the above property. This induction gives infinitely many $P_{n}$ for $n \in \omega$ all $\alpha$-equivalent but pairwise nonisomorphic. Without loss of generality we may assume that none of them is isomorphic to $T$. Now we can further code reals into the models. Enumerate the elements of $M(S)$ by $u_{0}, u_{1}, \ldots$, and denote the sequence by $\vec{u}$. For any subset $A$ of $\omega$, let $Q_{i}=P_{i}$ if $i \in A$ and $Q_{i}=T$ otherwise. Let $\vec{Q}$ denote the sequence $Q_{0}, Q_{1}, \ldots$ Then define $P_{A}=\Upsilon(\mathbb{A}, \vec{u}, \vec{Q})$. Apparently for $A \neq A^{\prime}$ we have $S_{A} \not S_{A^{\prime}}$. Thus there are $2^{\aleph_{0}}$ many models $\alpha$-equivalent to $T$ but pairwise nonisomorphic.

In this situation it is easy to construct $\mathbb{B}$ with $\mathbb{B} \equiv_{\alpha} \mathbb{A}$ but $\mathbb{B} \not \mathbb{A}$. Since $\mathbb{A}$ is countable, there must be some $A \subseteq \omega$ such that $S_{A} \hookrightarrow A$. Now obtain $\mathbb{B}$ from $\mathbb{A}$ by substituting any copy of $T$ in $\mathbb{A}$ by $S_{A}$. Then $S_{A} \hookrightarrow \mathbb{B}$ and therefore $\mathbb{B} \not \mathbb{A}$. That $\mathbb{B} \equiv \equiv_{\alpha} \mathbb{A}$ is obvious.

More generally, if there exists any tree $P$ with $P \equiv_{\alpha} T$ and $M(P)$ infinite the above construction finishes the proof. Thus for the rest of the proof we suppose that $M(P)$ is finite for all $P \equiv_{\alpha} T$. This implies that, for any $P$ with $P \equiv_{\alpha} T$ and any $l \in \omega$, there are only finitely many $u \in P$ with $P(u) \equiv_{\alpha} T$ and $\operatorname{lh}(u)=l$.

Next we analyze the structure of $\mathbb{A}$. Let $N(\mathbb{A})=\left\{y \in \mathbb{A} \mid \mathbb{A}(y) \equiv_{\alpha} T\right\}$. If $N(\mathbb{A})$ is well-founded, then we can let $M(\mathbb{A})$ be the set of all minimal elements of $N(\mathbb{A})$ and obtain $\mathbb{B}_{1}=\Upsilon(\mathbb{A}, M(\mathbb{A}), S)$ and $\mathbb{B}_{2}=\Upsilon(\mathbb{A}, M(\mathbb{A}), T)$. Then $\mathbb{B}_{1} \equiv_{\alpha} \mathbb{B}_{2} \equiv_{\alpha} \mathbb{A}$ but $\mathbb{B}_{1} \nsucceq \mathbb{B}_{2}$; thus one of them is not isomorphic to $\mathbb{A}$. Let $\mathbb{B}=\mathbb{B}_{1}$ if $\mathbb{B}_{1} \nsucceq \mathbb{A}$ and $\mathbb{B}=\mathbb{B}_{2}$ otherwise. Then $\mathbb{B}$ is as required. 
Thus for the rest of the proof we assume that $N(\mathbb{A})$ is ill-founded. We consider two further subcases.

Subcase 4.1 There are incomparable elements in $N(\mathbb{A})$.

Let $x_{0}$ and $y_{0}$ be two incomparable elements of $N(\mathbb{A})$. Let $x_{1}, x_{2}, \ldots$ enumerate the set $\left\{f^{l}\left(x_{0}\right) \mid l>0\right\} \cap N(\mathbb{A})$ in decreasing order. By our assumption, $x_{n}$ is defined for all $n \in \omega$. Suppose

$$
\forall n \in \omega \exists y_{n} \in N(\mathbb{A})\left(y_{n} \perp x_{n}\right) .
$$

Let

$$
Y=\left\{y \in \mathbb{A} \mid \exists n \in \omega\left(x_{n}<y \wedge y \in M\left(\mathbb{A}\left(x_{n}\right)\right) \wedge y \neq x_{n-1}\right)\right\} .
$$

Then $Y$ is the set of minimal elements in $M(\mathbb{A})$ incomparable with some $x_{n}$. Note that elements in $Y$ are pairwise incomparable. Now we can obtain $\mathbb{B}_{1}=\Upsilon(\mathbb{A}, Y, S)$ and $\mathbb{B}_{2}=\Upsilon(\mathbb{A}, Y, T)$. Then $\mathbb{B}_{1} \equiv_{\alpha} \mathbb{B}_{2}$. As to the isomorphism type, $\mathbb{B}_{1}$ satisfies the property that

$$
\begin{aligned}
\forall z \in \mathbb{B}_{1} \exists k>0 \forall l>k\left(f^{l}(z) \in\right. & N\left(\mathbb{B}_{1}\right) \Rightarrow \\
& \left.\forall y \in M\left(\mathbb{B}_{1}\left(f^{l}(z)\right)\right)\left(y \not \leq z \Rightarrow \mathbb{B}_{1}(y) \cong S\right)\right),
\end{aligned}
$$

while $\mathbb{B}_{2}$ satisfies the similar property with the occurrence of $S$ replaced by $T$. However, under the hypothesis (1) these two properties are contradictory. Thus $\mathbb{B}_{1} \nsucceq \mathbb{B}_{2}$ and one of them is not isomorphic to $\mathbb{A}$.

Now we suppose the hypothesis (1) fails. That is, suppose that there is some $n_{0}$ such that $x_{n_{0}}$ is comparable with all elements of $N(\mathbb{A})$. Without loss of generality assume $n_{0}=1$. Then for all $y \in N(\mathbb{A})$, either $y \geq x_{1}$ or $y=x_{n}$ for some $n$. Thus $x_{1}$ is the smallest element of $N(\mathbb{A})$ so that $\left|M\left(\mathbb{A}\left(x_{1}\right)\right)\right|>1$. Now we define $\mathbb{B}_{1}=\Upsilon\left(\mathbb{A}, M\left(\mathbb{A}\left(x_{1}\right)\right), \mathbb{A}\left(x_{1}\right)\right)$ and $\mathbb{B}_{2}=\Upsilon\left(\mathbb{A}, M\left(\mathbb{A}\left(x_{1}\right)\right), \mathbb{A}\left(x_{2}\right)\right)$. Then in both $\mathbb{B}_{1}$ and $\mathbb{B}_{2}, x_{1}$ has the same property as before; thus any isomorphism between $\mathbb{B}_{1}$ and $\mathbb{B}_{2}$ must leave it fixed. However, in $\mathbb{B}_{1}$ it happens that

$$
\forall y \in M\left(\mathbb{B}_{1}\left(x_{1}\right)\right)\left|M\left(\mathbb{B}_{1}(y)\right)\right|=1,
$$

whereas $\mathbb{B}_{2}$ satisfies that

$$
\forall y \in M\left(\mathbb{B}_{2}\left(x_{1}\right)\right)\left|M\left(\mathbb{B}_{2}(y)\right)\right|>1 .
$$

Thus $\mathbb{B}_{1} \neq \mathbb{B}_{2}$ and one of them is not isomorphic to $\mathbb{A}$. This finishes our proof of Subcase 4.1.

Subcase 4.2 $N(\mathbb{A})$ is linearly ordered.

Since $S \hookrightarrow T \hookrightarrow \mathbb{A}$ it follows that $N(S)$ and $N(T)$ are linearly ordered too. Let $x_{0} \in S$ be a minimal element with $S\left(x_{0}\right) \cong T$ and let $l=\operatorname{lh}\left(x_{0}\right)$. Let $y_{0} \in \mathbb{A}$ with $\mathbb{A}\left(y_{0}\right) \cong S$. Then for every $y \geq y_{0}$ with $\mathbb{A}(y) \equiv_{\alpha} T$ there is $z_{1} \geq z_{2} \geq y$ with $f^{l}\left(z_{1}\right)=z_{2}$ and $\mathbb{A}\left(z_{1}\right) \neq \mathbb{A}\left(z_{2}\right)$. Thus $\mathbb{A}$ satisfies the following property:

$$
\begin{aligned}
\exists y_{0} \in N(\mathbb{A}) \forall y & \geq y_{0}(y \in N(\mathbb{A}) \Rightarrow \\
& \left.\exists z_{1}, z_{2} \geq b\left(z_{1}, z_{2} \in N(\mathbb{A}) \wedge f^{l}\left(z_{1}\right)=z_{2} \wedge \mathbb{A}\left(z_{1}\right) \varsubsetneqq \mathbb{A}\left(z_{2}\right)\right)\right) .
\end{aligned}
$$

Now we use the tree $S_{\omega}$ constructed in Section 4 again. It is also true that $N\left(S_{\omega}\right)$ is linearly ordered. Moreover, it happens that $S_{\omega}$ satisfies that

$$
\forall b \in N\left(S_{\omega}\right) \exists a \geq b\left(a \in N(\mathbb{A}) \wedge f^{l}(a)=b \wedge S_{\omega}(a) \cong S_{\omega}(b)\right) .
$$


Define $\mathbb{B}=\Upsilon\left(\mathbb{A}, y_{0}, S_{\omega}\right)$. Then $\mathbb{B}$ satisfies that

$$
\forall b \in N(\mathbb{B}) \exists c_{1}, c_{2} \geq b\left(c_{1}, c_{2} \in N(\mathbb{B}) \wedge f^{l}\left(c_{1}\right)=c_{2} \wedge \mathbb{B}\left(c_{1}\right) \cong \mathbb{B}\left(c_{2}\right)\right) .
$$

This shows that $\mathbb{B} \nsucceq \mathbb{A}$. Since $\mathbb{B} \equiv_{\alpha} \mathbb{A}$, we have proved the main theorem.

\section{References}

[1] Barwise, J., Admissible Sets and Structures. An Approach to Definability Theory, Perspectives in Mathematical Logic, Springer-Verlag, Berlin, 1975. Zbl 0316.02047. MR 0424560. 34, 36

[2] Becker, H., and A. S. Kechris, The Descriptive Set Theory of Polish Group Actions, vol. 232 of London Mathematical Society Lecture Note Series, Cambridge University Press, Cambridge, 1996. Zbl 0781.03033. MR 1425877. 33

[3] Gao, S., "A dichotomy theorem for mono-unary algebras," Fundamenta Mathematicae, vol. 163 (2000), pp. 25-37. Zbl 0955.03049. MR 1750333. 34

[4] Gao, S., "Some dichotomy theorems for isomorphism relations of countable models," The Journal of Symbolic Logic, vol. 66 (2001), pp. 902-22. Zbl 0992.03057. MR 1833486. 34, 37

[5] Harrington, L. A., A. S. Kechris, and A. Louveau, "A Glimm-Effros dichotomy for Borel equivalence relations," Journal of the American Mathematical Society, vol. 3 (1990), pp. 903-28. Zbl 0778.28011. MR 1057041. 33

[6] Hjorth, G., “A dichotomy for isomorphism,” unpublished manuscript. 34

[7] Kechris, A. S., and D. A. Martin, "Infinite games and effective descriptive set theory," pp. 404-70 in Analytic Sets, edited by C. A. Rogers et al., Academic Press Inc. [Harcourt Brace Jovanovich Publishers], London, 1980. Lectures delivered at a Conference held at University College, University of London, July 16-29, 1978. Zbl 0451.04001. MR 608794. 33

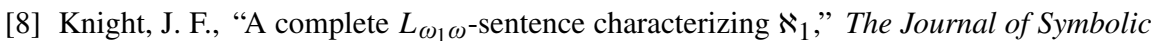
Logic, vol. 42 (1977), pp. 59-62. Zbl 0426.03037. MR 0491141. 36

[9] Makkai, M., “An example concerning Scott heights," The Journal of Symbolic Logic, vol. 46 (1981), pp. 301-18. Zbl 0501.03018. MR 613284. 34, 36

[10] Marcus, L., "The number of countable models of a theory of one unary function," Fundamenta Mathematicae, vol. 108 (1980), pp. 171-81. Zbl 0363.02055. MR 594316. 34

[11] Miller, A. W., "Vaught's conjecture for theories of one unary operation," Fundamenta Mathematicae, vol. 111 (1981), pp. 135-41. Zbl 0492.03012. MR 609429. 34

[12] Rubin, M., "Vaught's conjecture for linear orderings," Notices of American Mathematical Society, vol. 24 (1977), A390. 34

[13] Steel, J. R., “On Vaught's conjecture," pp. 193-208 in Cabal Seminar 76-77 (Proceedings of the Caltech-UCLA Logic Seminar, 1976-77), vol. 689 of Lecture Notes in Mathematics, Springer, Berlin, 1978. Zbl 0403.03027. MR 526920. 34, 36, 37 


\section{Acknowledgments}

Based on a talk in the Workshop on Classification of Countable Models, University of Notre Dame, May 18-24, 2005. Research supported by the U.S. NSF grant DMS0501039. I would like to thank the entire logic group at the University of Notre Dame for the organization of the Workshop on Classification of Countable Models. More specifically I thank Peter Cholak and Julia Knight for discussions on the subject of this article and for the encouragement to write it up. I would also like to thank the participants of the workshop for useful discussions on the topic and, especially, Greg Hjorth for his generosity to share Proposition 3.1 with me and the permission to include it here.

Department of Mathematics

University of North Texas

PO Box 311430

Denton TX 76203

sgao@unt.edu 